\title{
UTILIZAÇÃo DE REGRESS̃̃O MULTIVARIADA PARA AVALIAÇÃO ESPECTROFOTOMÉTRICA DA DEMANDA QUÍMICA DE OXIGÊNIO EM AMOSTRAS DE RELEVÂNCIA AMBIENTAL
}

\author{
Patricio Peralta-Zamora* e Gilcélia A. Cordeiro \\ Departamento de Química, Universidade Federal do Paraná, CP 19081, 81531-990 Curitiba-PR \\ Noemi Nagata \\ Departamento de Química, Universidade Estadual de Ponta Grossa, Campus de Uvaranas, 84030-900 Ponta Grossa-PR
}

Recebido em 31/8/04; aceito em 23/2/05; publicado na web em 30/6/05

\begin{abstract}
USE OF MULTIVARIATE REGRESSION IN SPECTROPHOTOMETRIC EVALUATION OF CHEMICAL OXIGEN DEMAND
IN SAMPLES OF ENVIRONMENTAL RELEVANCE. In this work, a partial least squares regression routine was used to develop a multivariate calibration model to predict the chemical oxygen demand (COD) in substrates of environmental relevance (paper effluents and landfill leachates) from UV-Vis spectral data. The calibration models permit the fast determination of the COD with typical relative errors lower by $10 \%$ with respect to the conventional methodology.
\end{abstract}

Keywords: COD; multivariate regression; spectrophotometry.

\section{INTRODUÇÃO}

Nos últimos anos, o recrudescimento dos problemas ambientais relacionados com as atividades humanas (domésticas e industriais) praticamente tem obrigado a criação de programas de vigilância, de maneira a se evitar que o elevado potencial poluente dos resíduos, assim como seu tratamento e sua disposição inadequadas levem à geração de problemas ambientais de difícil recuperação. Dentro deste contexto, percebe-se que a legislação tende a ficar cada vez mais restritiva, enquanto que a fiscalização se torna mais presente.

Dentre as várias ferramentas utilizadas para avaliar o potencial poluente de um resíduo, pode ser dado destaque à Demanda Química de Oxigênio (DQO), parâmetro que reflete grande parte da matéria orgânica presente na matriz e que, portanto, aparece em grande parte das legislações ambientais do mundo ${ }^{1}$.

Embora se trate de um parâmetro consolidado e utilizado mundialmente como critério de avaliação, sua determinação apresenta sérios inconvenientes, principalmente em função dos seguintes aspectos: o procedimento padrão ${ }^{2}$ fundamenta-se na oxidação da matéria orgânica pelo dicromato de potássio, em meio sulfúrico e a elevadas temperaturas, o que implica sérios riscos de acidentes; envolve o uso de catalisadores, usualmente de preço elevado $\left(\mathrm{Ag}_{2} \mathrm{SO}_{4}\right)$; envolve a geração de resíduos contendo cromo e mercúrio, o que faz com que seu caráter poluente seja elevado; envolve uma etapa de digestão que demora em torno de $2 \mathrm{~h}$, o que acarreta tempo de análise elevado e, a reprodutibilidade da técnica costuma ser bastante baixa, freqüentemente levando a desvios de até $30 \%{ }^{3}$.

Em função destes antecedentes, torna-se interessante desenvolver uma alternativa analítica que permita a avaliação deste parâmetro, por meio de procedimentos mais simples, rápidos e seguros. As experiências acumuladas ao longo de vários anos de trabalho na área de tratamento de resíduos nos permitiram verifi-

*e-mail: zamora@quimica.ufpr.br car a existência de elevada correlação entre a DQO e o sinal espectrofotométrico apresentado pelos diversos resíduos estudados, principalmente quando considera a área espectral integrada entre 200 e $700 \mathrm{~nm}$. Este argumento, junto com dois casos de sucesso reportados na literatura ${ }^{4,5}$, leva-nos a propor a utilização de regressão de mínimos quadrados parciais $\left(\mathrm{PLSR}^{6}\right)$ para o desenvolvimento de modelos multivariados destinados à determinação de DQO em matrizes de relevância ambiental, utilizandose espectroscopia UV-Vis.

\section{PARTE EXPERIMENTAL}

Resíduos papeleiros (efluentes de polpação e de branqueamento) foram gentilmente cedidos por Klabin S.A. (Telêmaco BorbaPR), enquanto que chorume de aterro sanitário foi doado pelo Aterro Municipal da Cachimba (Curitiba-PR).

Os resíduos foram submetidos a diversos processos avançados de degradação (fotocatálise heterogênea e peroxidação assistida por radiação ultravioleta), aplicados de acordo com procedimentos descritos previamente ${ }^{7,8}$. Para o efluente papeleiro de branqueamento 30 amostras foram produzidas, 11 das quais foram reservadas para calibração e 19 para validação. Nos estudos envolvendo efluente de polpação, o modelo foi desenvolvido com 12 amostras, sendo outras 18 reservadas para validação. Finalmente, o trabalho com chorume de aterro sanitário envolveu o preparo de 36 amostras, 20 das quais foram utilizadas para calibração.

As determinações de DQO foram realizadas de acordo com procedimento padrão ${ }^{2}$, que consistente em digestão em tubo fechado, seguida de determinação espectrofotométrica.

Os espectros foram registrados entre 200 e $700 \mathrm{~nm}$, em espectrofotômetro SCINCO S-1000, utilizando-se cubetas de $1 \mathrm{~cm}$.

Os modelos multivariados foram desenvolvidos por regressão de mínimos quadrados parciais, utilizando-se o programa PLStoolbox 1.5, que opera em ambiente Matlab 4.0. Os espectros foram processados entre 199 e $401 \mathrm{~nm}$ (150 pontos), sendo previamente centrados na média. Para determinação do melhor número de variáveis latentes utilizou-se uma rotina de "cross validation" (sistema "leave-one out") . 


\section{RESULTADOS E DISCUSSÃO}

Em primeiro lugar é importante salientar que, durante vários anos de trabalho na área de degradação de resíduos, uma importante correlação foi observada entre a evolução do perfil espectroscópico e da DQO. Esta observação, que é comum para resíduos papeleiros e têxteis e para outras matrizes de relevância ambiental (ex., chorume de aterro sanitário, águas contaminadas com derivados de petróleo, etc.), pode ser verificada a partir dos antecedentes apresentados na Figura 1. Durante a degradação de efluente papeleiro de polpação, o perfil espectral modifica-se sensivelmente, sendo acompanhado por modificações proporcionais da DQO (r: 0,976). Pequenas inconsistências provavelmente sejam devidas à presença dos agentes oxidantes utilizados no processo de degradação (ex., $\mathrm{H}_{2} \mathrm{O}_{2}$ ) e à irreprodutibilidade que caracteriza a determinação de DQO.

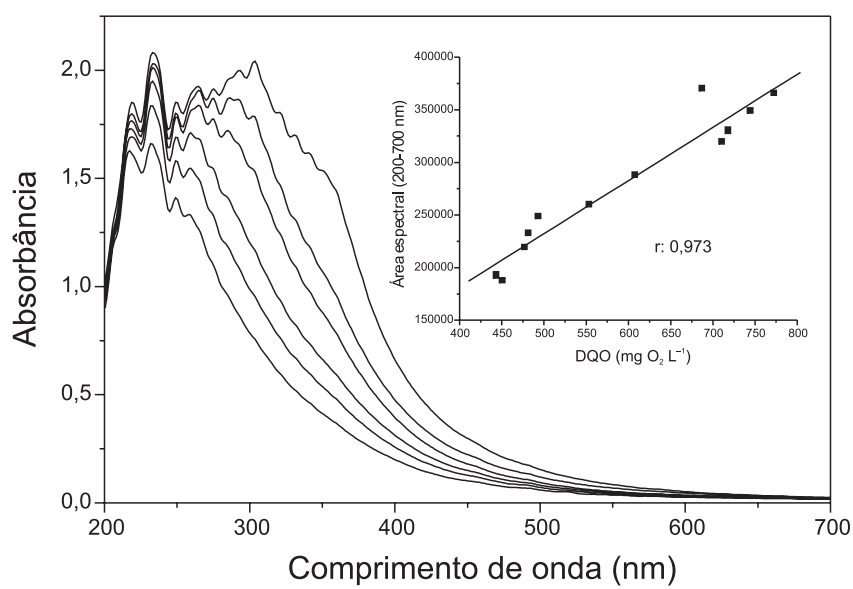

Figura 1. Evolução do espectro UV-Vis durante a degradação de efluente papeleiro de polpação e correlação entre área espectral integrada (200-700 nm) e $D Q O$

Em função destes argumentos, a proposta de se utilizar espectroscopia UV-Vis para avaliação da DQO é bastante consistente, desde que um critério fundamental seja respeitado, isto é, a avaliação espectroscópica da DQO somente pode ser realizada a partir de modelos de calibração desenvolvidos com o próprio substrato em estudo. Por motivos óbvios, modelos espectroscópicos universais não podem ser desenvolvidos, uma vez que perfis espectroscópicos similares podem representar valores de DQO completamente diferenciados.

Embora modelos multivariados tenham sido elaborados para uma grande variedade de substratos, apenas um estudo de desenvolvimento será apresentado, envolvendo efluente papeleiro de branqueamento. Na primeira etapa, o valor de PRESS (somatória do quadrado dos erros de previsão) obtido no processo de validação cruzada (Figura 2) pode ser utilizado como argumento para a escolha do número de variáveis latentes (VL) necessário para elaboração do modelo multivariado de calibração. Embora o mínimo PRESS seja obtido com a utilização de $10 \mathrm{VL}$, pode-se observar que as 2 primeiras VLs descrevem aproximadamente $98 \%$ da variância dos resultados de DQO, utilizando praticamente $99 \%$ dos dados espectrais. Em função deste fato, e do valor de PRESS apresentar um mínimo para 2 VLs, o modelo foi elaborado com apenas duas variáveis latentes. Em geral, a utilização de um número excessivo de VLs pode levar a uma situação de super-ajuste do modelo, fazendo com que algumas VLs sejam utilizadas, apenas, para descrever anomalias apresentadas pelo conjunto de calibração ou ruídos instrumentais.

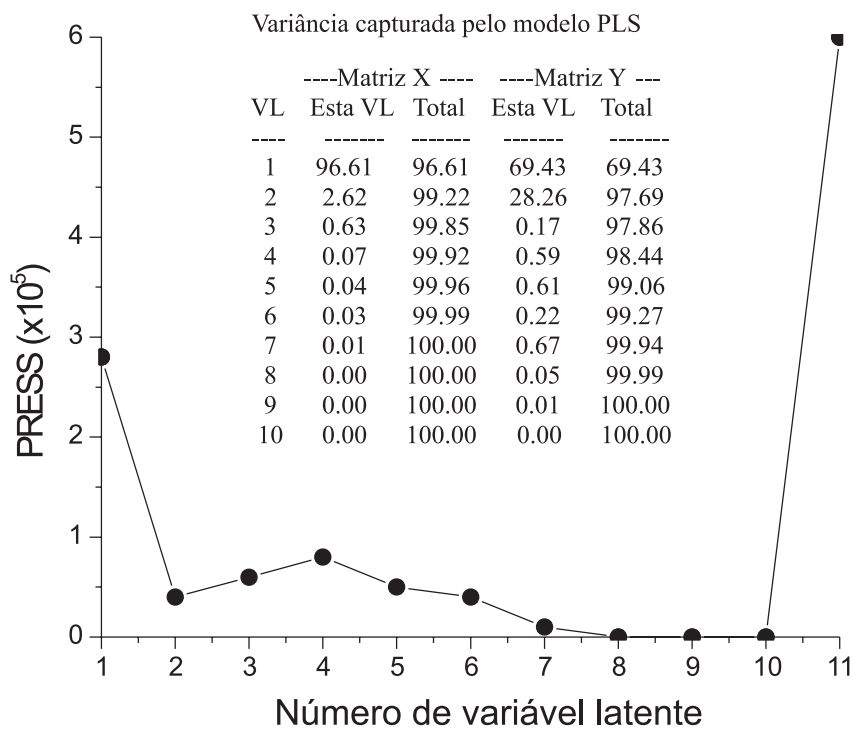

Figura 2. Relação entre o número de variáveis latentes e a somatória do quadrado dos erros de previsão, para modelo desenvolvido para efluente de branqueamento

Utilizando-se os resíduos de Student e Leverage como critérios de detecção de anomalias (Figura 3), é possível perceber que nenhum dos padrões do conjunto de calibração apresenta valores que extrapolam os limites de 2,5 e 0,54 (3 VL/N), definidos como valores máximos para resíduos e "leverage", respectivamente. A detecção de "outliers" é uma importante ferramenta adicional que o sistema de calibração multivariada disponibiliza, evitando a inclusão de argumentos que viciem os modelos.

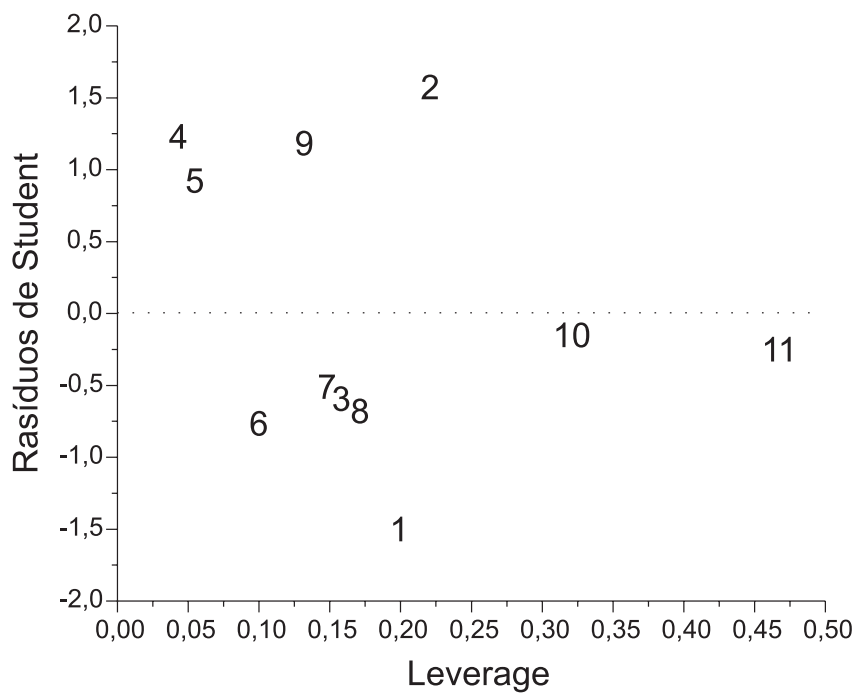

Figura 3. Resíduos de Student vs Leverage, para modelo desenvolvido para efluente de branqueamento (2 VLS)

O excelente ajuste do modelo pode ser visualizado no gráfico de valores previstos por valores reais, apresentado na Figura 4. De maneira geral, é possível observar que a informação contida nos espectros UV-Vis pode ser representada por duas variáveis latentes, a partir das quais é possível se desenvolver um modelo de regressão que se correlaciona adequadamente com os valores de DQO.

Para verificar a validade dos modelos (validação), o valor da DQO de novas amostras foi previsto a partir dos respectivos espectros, dando lugar aos resultados apresentados na Tabela 1. De maneira geral, observa-se que os modelos desenvolvidos para efluente 


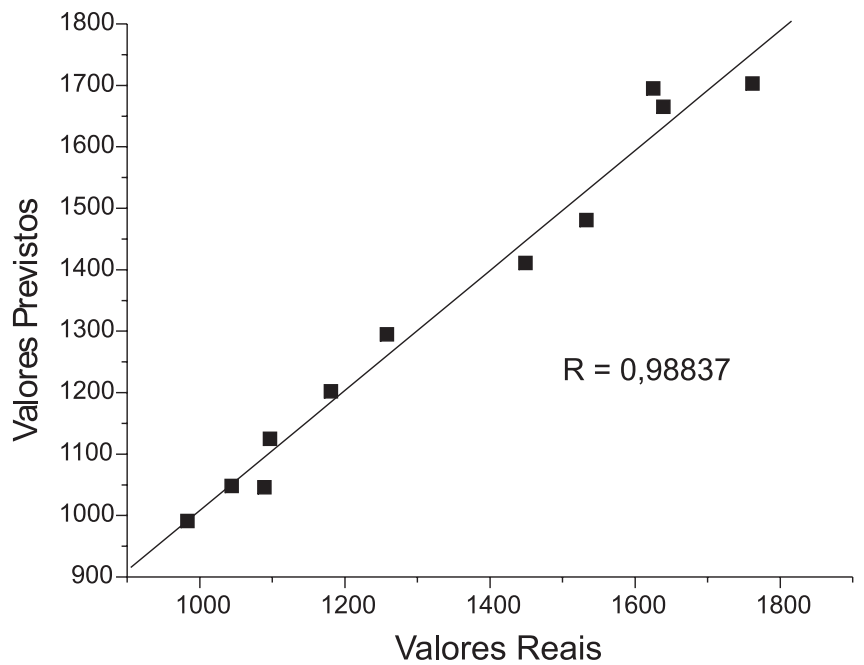

Figura 4. Valores reais vs valores previstos, para modelo desenvolvido para efluente de branqueamento ( $2 \mathrm{VLs}$ )

de branqueamento (2 VLs), efluente de polpação (3 VLs) e chorume de aterro sanitário (3 VLs) permitiram a obtenção de resultados bastante coerentes com seus análogos obtidos pela metodologia convencional, com erros relativos máximos da ordem de $15 \%$. Atribuir discrepâncias desta magnitude é bastante difícil, principalmente levando-se em consideração a baixa reprodutibilidade da metodologia de referência. Entretanto, cabe ressaltar que a metodologia convencional citada apresenta elevada tendência a erros po- sitivos, por conta da interferência de espécies inorgânicas que podem ser oxidadas pelo dicromato.

Em função das dificuldades envolvidas na metodologia convencional já comentadas, novas propostas têm surgido para a rápida avaliação deste parâmetro. Dentre outros trabalhos destacam-se sistemas de digestão tradicional seguidos de avaliação por quimioluminiscência ${ }^{10}$ ou sistemas de digestão modificados, fundamentados, por ex., em fotocatálise heterogênea ${ }^{11}$ e ultrasom ${ }^{12}$. Dentro deste contexto destaca-se, ainda, a proposta de um sistema miniaturizado $^{3}$, que reduz bastante a geração de resíduos. Em todos estes casos, foram reportados erros ou desvios relativos superiores aos aqui apresentados.

\section{CONCLUSÕES}

No decorrer deste trabalho verificou-se que, tal como esperado, existe uma consistente correlação entre o sinal espectroscópico registrado na região UV-Vis e o teor de matéria orgânica, representado pela demanda química de oxigênio, para diversos resíduos de relevância ambiental. A informação contida nos espectros pode ser convenientemente representada por um conjunto de poucas variáveis latentes, o que facilita o desenvolvimento de um modelo de regressão multivariado, fundamentado em regressão de mínimos quadrados parciais (PLSR). Os modelos desenvolvidos mostramse robustos, permitindo a determinação da DQO em amostras de efluentes papeleiros e chorume de aterro sanitário, com baixos erros relativos em relação à metodologia padrão. Trata-se de um resultado relevante, uma vez que a simplicidade e rapidez da metodologia proposta poderiam permitir a implementação de sis-

Tabela 1. Resultados de DQO previstos pelos modelos multivariados para amostras de efluente de branqueamento, polpação e chorume, submetidas a processos de degradação

\begin{tabular}{|c|c|c|c|c|c|c|c|c|c|}
\hline & \multicolumn{3}{|c|}{$\begin{array}{l}\text { Efluente papeleiro } \\
\text { de branqueamento }\end{array}$} & \multicolumn{3}{|c|}{$\begin{array}{l}\text { Efluente papeleiro } \\
\text { de polpação }\end{array}$} & \multicolumn{3}{|c|}{$\begin{array}{c}\text { Chorume de } \\
\text { aterro sanitário }\end{array}$} \\
\hline & Real & Previsto & Erro (\%) & Real & Previsto & Erro $(\%)$ & Real & Previsto & Erro (\%) \\
\hline \multirow[t]{4}{*}{ Processo 1} & 1632 & 1669 & $+2,26$ & 686 & 764 & $+11,31$ & 5061 & 4714 & $-6,86$ \\
\hline & 1701 & 1669 & $-1,87$ & 778 & 766 & $-1,58$ & 4120 & 4174 & $+1,31$ \\
\hline & 1250 & 1180 & $-5,58$ & 617 & 588 & $-4,80$ & 3908 & 3774 & $-3,43$ \\
\hline & 1051 & 1002 & $-4,73$ & 490 & 446 & $-9,04$ & 3840 & 3799 & $-1,07$ \\
\hline \multirow[t]{14}{*}{ Processo 2} & 1586 & 1633 & $+2,92$ & 667 & 761 & $+14,08$ & & & \\
\hline & 1564 & 1573 & $+0,63$ & 690 & 756 & $+9,57$ & & & \\
\hline & 1418 & 1447 & $+1,99$ & 652 & 732 & $+12,27$ & & & \\
\hline & 1426 & 1341 & $-5,95$ & 671 & 709 & $+5,65$ & & & \\
\hline & 1476 & 1273 & $-13,74$ & 652 & 684 & $+4,94$ & 4620 & 4908 & $+6,23$ \\
\hline & 1334 & 1232 & $-7,68$ & 602 & 656 & $+8,99$ & 4009 & 3437 & $+14,27$ \\
\hline & 1045 & 1037 & $-0,79$ & 587 & 635 & $+8,26$ & 4840 & 5249 & $+8,45$ \\
\hline & & & & 586 & 610 & $+4,05$ & 4091 & 3668 & $-10,34$ \\
\hline & & & & 556 & 590 & $+6,12$ & 3960 & 3447 & $-12,95$ \\
\hline & & & & 579 & 543 & $-6,26$ & 3122 & 1928 & $-38,24$ \\
\hline & & & & 571 & 509 & $-10,85$ & & & \\
\hline & & & & 526 & 483 & $-8,09$ & & & \\
\hline & & & & 507 & 458 & $-9,61$ & & & \\
\hline & & & & 472 & 445 & $-5,79$ & & & \\
\hline \multirow[t]{8}{*}{ Processo 3} & 1678 & 1637 & $-2,45$ & & & & & & \\
\hline & 1508 & 1324 & $-12,23$ & & & & 5680 & 3911 & $-31,14$ \\
\hline & 1288 & 1212 & $-5,92$ & & & & 5460 & 4816 & $-11,79$ \\
\hline & 1265 & 1106 & $-12,61$ & & & & 5320 & 5100 & $-4,13$ \\
\hline & 1135 & 1028 & $-9,46$ & & & & 4180 & 4814 & $+15,17$ \\
\hline & 1112 & 1095 & $-1,55$ & & & & 4410 & 4829 & $+9,50$ \\
\hline & 1005 & 1075 & $+6,90$ & & & & 3301 & 2898 & $-12,21$ \\
\hline & 990 & 1027 & $+3,70$ & & & & & & \\
\hline
\end{tabular}


temas de monitoramento "on line", tão necessários em estações de tratamento de resíduos, por ex.

\section{AGRADECIMENTOS}

Agradecemos as colaborações das alunas de Doutorado J. L. de Morais e de Iniciação Científica L. M. Carneiro, por fornecerem os dados necessários para a elaboração deste trabalho.

\section{REFERÊNCIAS}

1. http://www.lei.adv.br/conama01.htm, acessada em Agosto 2004.

2. APHA, Standard Methods for Examination of Water and Wastewater, $19^{\text {th }}$ ed., Amer. Publ. Ass: Washington, 1995.

3. LaPara, T. M.; Alleman, J. E.; Pope, P. G.; Waste Manage. 2000, 20, 295.
4. Langergraber, G.; Fleischmann, N.; Hofstadter, F.; Water Sci. Technol. 2003, 47, 63.

5. Eriksson, L.; Hagberg, P.; Johansson, E.; Rannar, S.; Whelehan, O.; Astrom, A.; Lindgren, T.; J. Chemom. 2001, 15, 337.

6. Ferreira, M. M. C.; Antunes, A. M.; Melgo, M. S.; Volpe, P. L. O.; Quim. Nova 1999, 22, 724

7. Peralta-Zamora, P. G.; Gomes de Moraes, S.; Pelegrini, R.; Freire Jr., M.; Reyes, J.; Mansilla, H.; Duran, N.; Chemosphere 1998, 36, 2119.

8. Kunz, A.; Peralta-Zamora, P. G.; Duran, N.; Adv. Environ. Res. 2002, 7, 197.

9. Martens, H.; Naes, T.; Multivariate Calibration, John Wiley \& Sons: Chichester, England, 1989.

10. Hu, Y.; Yang, Y.; Talanta 2004, 63, 521

11. Ai, S.; Li, J.; Yang, Y.; Gao, M.; Pan, Z.; Jin, L.; Anal. Chim. Acta 2004, 509, 237.

12. Canals, A,; Cuesta, A.; Gras, L.; Hernández, M. R.; Ultrason. Sonochem. 2002, 9, 143. 